\title{
Second by second patterns in cortical electroencephalograph and systolic blood pressure during Cheyne-Stokes
}

\author{
R.J.O. Davies*, L.S. Bennet*, C. Barbour*, L. Tarassenko**, J.R. Stradling*
}

Second by second patterns in cortical electroencephalograph and systolic blood pressure during Cheyne-Stokes breathing. R.J.O. Davies, L.S. Bennet, C.Barbour, L. Tarassenko, J.R. Stradling. (C) ERS Journals Ltd 1999.

ABSTRACT: Little is known about how arousal develops during the ventilatory phase of Cheyne-Stokes breathing. This study employs neural network analysis of electroencephalograms (EEGs) to describe these changes and relate them to changes in systolic blood pressure, which is probably a subcortical marker of arousal.

Six patients with Cheyne-Stokes respiration (apnoea/hypopnoea index 32-69 $h^{-1}$ ) caused by stable chronic heart failure underwent polysomnography including arterial beat-to-beat systolic blood pressure determination. Periods of 15 sequential apnoeas during nonrapid eye movement sleep were identified for each subject. For each apnoea, the EEG was examined second-by-second using neural net analysis from $28 \mathrm{~s}$ before to $28 \mathrm{~s}$ after apnoea termination (first return of oronasal airflow), and this was compared with the systolic blood pressure pattern.

During the apnoeic phase, sleep deepened progressively. Arousal started to develop at or just before apnoea termination and progresses through the breathing phase. The rise and fall in the systolic blood pressure closely followed the rise and fall in electroencephalographic sleep depth.

In conclusion, during Cheyne-Stokes breathing, cortical electroencephalographic arousal begins at or just before the resumption of breathing. Cortical electroencephalographic sleep depth changes are closely mirrored by changes in arterial systolic blood pressure, suggesting that the state changes in the cortical and basal brain structures may be synchronous.

Eur Respir J 1999; 14: 940-945.

Cheyne-Stokes breathing is a common disorder of breathing during sleep. It affects up to $50 \%$ of patients with chronic heart failure $[1,2]$ and can cause sufficient sleep fragmentation through recurrent arousals to produce a daytime sleep deprivation syndrome [3,4]. Detailed study of this sleep fragmentation is difficult using standard sleep scoring techniques. Standard epoch-based scoring [5] resolves the sleep process to only 20-30 s which is an inadequate way of quantifying a continuously varying process in which a whole apnoea/breathing cycle can occur in $>60$ s. Even newer approaches to counting recurrent arousals from sleep only describe the number of events exceeding an arbitrary threshold (usually $3 \mathrm{~s}$ duration [6]). These methods give no information about the dynamics of the arousal process and neglect all events that are below the selected threshold even though very minor arousal events can cause significant functional daytime impairment [7].

The limitations of current standard sleep scoring techniques mean that little detail is known of how cortical electroencephalographic arousal progresses and regresses during sleep in breathing disorders such as Cheyne-Stokes breathing. The temporal relationships between electroencephalographic features of arousal and autonomic features of brainstem activation blood pressure elevation, cardioacceleration, skin vasoconstriction, etc. [8]) are also poorly described. In obstructive sleep apnoea there is some
*Osler Chest Unit, Churchill Hospital Site, Oxford Radcliffe Hospital, Headington, Oxford, UK. OX3 7LJ. **Dept of Medical Engineering, Oxford University, Parks Road, Oxford, UK.

Correspondence: R.J.O. Davies

Osler Chest Unit

Churchill Hospital Site

Oxford Radcliffe Hospital

Headington

Oxford

UK

Fax: 441865225221

Keywords: Arousal

Cheyne-Stokes respiration

electroencephalography

heart failure

sleep apnoea syndromes

Received: April 241998

Accepted after revision March 71999

This study was supported by the Wellcome Trust, Project Grant No. 046430. available information. A gradual shift in the electroencephalographic spectral power towards higher-frequency activity (and by inference wakefulness) has been reported late in apnoea, with a further shift towards higher frequencies after apnoea termination even if this phase is not associated with visual electroencephographic arousal $[9$, $10]$. There is also limited data relating to periodic breathing. Here, the waxing and waning respiratory cycle is associated with a rise in high-frequency electroencephalographic activity during the hyperpnoeic phase [11].

This study expands these observations by applying a novel method for quantifying the depth of nonrapid eye movement (non-REM) sleep on a second-by-second basis to the electroencephalogram (EEG) recorded during Cheyne-Stokes breathing. The results of this analysis are compared with the timing of the apnoea/breathing cycle and a nonelectroencephalographic marker of brain stem activation, systolic blood pressure.

\section{Methods}

\section{Subjects}

Six nonsnoring subjects with Cheyne-Stokes breathing caused by chronic heart failure were studied. The study was approved by the Central Oxford Research Ethics Committee and subjects gave consent in line with the committee's requirements. At the time of the study, none of 
the subjects with Cheyne-Stokes breathing exhibited clinical or chest radiographic evidence of fluid overload. All showed objective evidence of impaired left ventricular function (ejection fraction $<30 \%$ ) without significant valvular dysfunction. The clinical characteristics of the subjects are shown in table 1.

\section{Sleep studies}

Subjects underwent polysomnography during which the EEG (C3-A2), two channels of the electro-oculogram, chin electromyogram, oronasal airflow (thermistor), and ribcage and abdominal movements (inductive plethysmography) were monitored. Signals were recorded on an eight-channel tape recorder (MPA 2; Oxford Medical, Abingdon, UK). Digital mean arterial blood pressure was recorded in the middle finger of the left hand using a noninvasive plethysmographic method validated against invasive arterial line measurements [12] (Finapres; Ohmeda, Boulder, CO, USA; linked to an IBM - compatible personal computer and interpolated to a 1-s resolution). This blood pressure monitor was switched off for $5 \mathrm{~min}$ in each 30 min to avoid patient discomfort. All the physiological signals were recorded synchronously. During the sleep study, the laboratory was monitored using a video/audio system and a recording of this was kept for later inspection.

\section{Sleep staging and respiratory analysis}

Sleep was initially staged according to standard criteria [5]. Apnoea was defined as a fall in oronasal airflow to $<20 \%$ of baseline for $>10 \mathrm{~s}$, and hypopnoea as a fall to $<50 \%$ for the same period. Cheyne-Stokes breathing was defined as periods of repetitive central apnoea interspersed with periods of waxing and waning ventilation (fig. 1). Apnoea termination was defined as the onset of the first post-apnoeic breath on the oronasal airflow trace and this was used as the reference point for the detailed analysis.

\section{Selection of periods of Cheyne-Stokes breathing for analy-} sis

The sleep studies were reviewed and non-REM sleep periods during which the EEG, respiratory signals and systolic blood pressure were technically adequate were identified. Periods during which the hand bearing the Finapres moved or the systolic blood pressure trace showed finger twitch artefacts were excluded. Between two and four periods of 15 sequential apnoea/breathing cycles from across the night were then selected for detailed analysis.

\section{Arousal scoring}

The EEG during the ventilatory phase following the termination of each apnoea was scored for the presence or absence of a 3-s arousal according to American Sleep Disorder Association (ASDA) criteria. For each post-apnoea ventilatory phase, arousal was considered either present or absent, and, when one event was present, no further events were scored until the termination of the next apnoea. Thus, the number of respiratory events ending in traditional electroencephalographic arousal were quantified (table 1).

\section{Electroencephalographic signal analysis}

The raw electroencephalographic signal was processed using a neural network trained on a frequency-domain representation of the EEG, which was calculated every second (Quester; Oxford Medical). This representation is based on an autoregressive model of the EEG [13] which characterizes the dominant frequencies in the EEG for

Table 1. - Clinical and sleep study characteristics of the subjects studied

\begin{tabular}{lccccc}
\hline $\begin{array}{l}\text { Subject } \\
\text { No. }\end{array}$ & LVEF $\%$ & \multicolumn{2}{c}{ Chronic heart failure } & Polysomnography results & 3-s arousal \\
frequency* \%
\end{tabular}

*: frequency of apnoeas studied in detail followed by a visual 3-s electroencephalographic arousal according to American Sleep Disorders Association criteria [6]; ${ }^{+}$: dose varies with time; LVEF: left ventricular ejection fraction; ECHO: echocardiography; MUGA: multiple gated acquisition blood pool scan; non-REM: nonrapid eye movement; AHI: apnoea/hypopnoea index. 


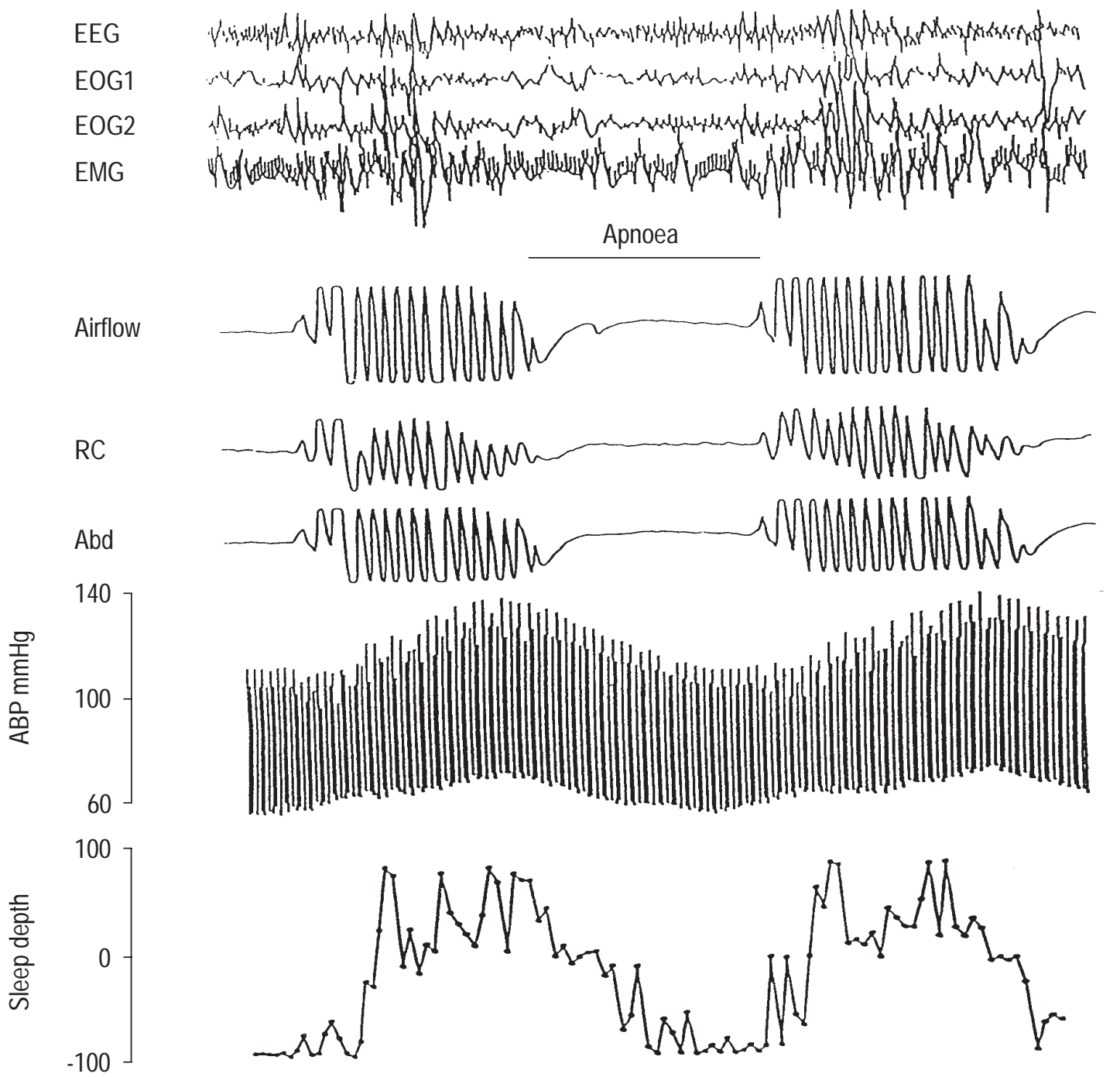

Fig. 1. - Cheyne-Stokes breathing showing recurrent apnoeas, recurrent electroencephalographic arousals, changes in sleep depth quantified from neural network analysis and post-apnoeic blood pressure rises. The apnoea marked lasted for approximately $30 \mathrm{~s}$. EEG: electroencephalogram; EOG: electro-oculogram; EMG: electromyogram; ABP: arterial blood pressure; RC: ribcage movement; Abd: abdominal movement.

each second. Consensus scored segments of EEGs corresponding to wakefulness, light rapid eye movement sleep and deep sleep drawn from normal subjects comprise the training database for the network. The advantage of this system is that the network, once trained, is able to interpolate between these states and hence track the sleep/ wake continuum without having to use the arbitrary categorical classifications of traditional sleep staging. A depth of sleep indicator is thus produced which describes sleep depth on a second-by-second basis and varies between $100 \%$ (totally awake) and $-100 \%$ (deeply asleep). A full description of this approach has been published elsewhere [13]. Examples of a raw EEG neural network second-bysecond output, systolic blood pressure and breathing pattern during Cheyne-Stokes breathing are shown in fig. 1.

For each apnoea, the depth of sleep from the neural network analysis was calculated for each of the $28 \mathrm{~s}$ before and after apnoea termination. These individual data series were averaged for each series of 15 apnoeas (fig. 2) and for each subject overall (fig. 3).

\section{Systolic blood pressure signal analysis}

Rises in systolic blood pressure are a marker of brainstem activation and arousal is one significant contributor to this response [8, 14], but represents brainstem rather than cortical activation. In order to correlate these changes with those of the cortical EEG during Cheyne-Stokes breathing, the systolic blood pressure for each of $28 \mathrm{~s}$ before and after apnoea termination was identified. These individual results were averaged for each series of 15 apnoeas to remove the effects of breathing - related blood pressure falls. These data were then presented as the mean for each sequence of 15 apnoeas (fig. 2) and for each subject overall (fig. 3).

\section{Results}

\section{Polysomnography and arousal scoring}

All the subjects exhibited typical Cheyne-Stokes breathing with apnoea/hypopnoea indices ranging 32-69 events. $\mathrm{h}^{-\mathrm{P}}$ In all subjects, the majority of the apnoeas studied in 


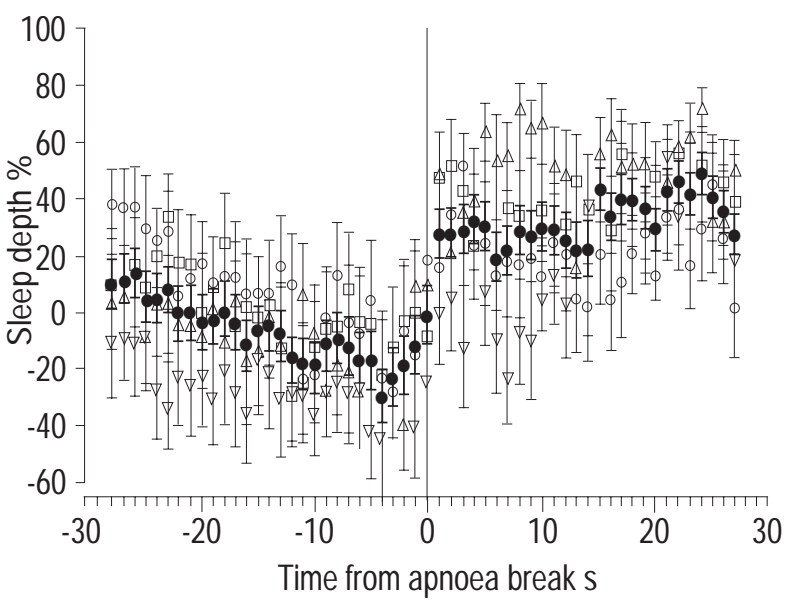

Fig. 2. - Detailed results from a representative subject (No. 3), showing the change in neural network depth of sleep averaged over four separate periods of 15 apnoeas caused by Cheyne-Stokes breathing (each represented by a different empty symbol), and the overall pattern of change $(\bullet)$. Data are presented as mean \pm SEM. The results show that the subject mean is representative of the individual data series. The onset of the first breath of the post-apnoeic ventilatory phase is indicated by the vertical bar. Sleep depth: $100 \%$ : totally awake; $-100 \%$ deeply asleep.

detail were followed by traditional 3-s electroencephalographic arousal during the post-apnoeic ventilatory phase. The frequency with which this occurred varied from 64 $97 \%$ of apnoeas (table 1).

\section{Neural network electroencephalographic and autonomic arousal patterns}

The relationships between the progression of cortical electroencephalographic arousal on neural network analysis, breathing and systolic blood pressure were surprisingly consistent. In all subjects, electroencephalographic sleep depth gradually increased throughout the apnoea, with the nadir in sleep depth occurring at or just before apnoea termination. Following apnoea termination, a change towards lighter sleep and "arousal" occurred in the postapnoeic ventilatory period in all subjects. This pattern was consistent both within the subjects across the night (fig. 2) and between subjects (fig. 3). One subject (No. 4) could not tolerate the Finapres device (fig. 3).

The systolic blood pressure changes were also consistent within and between subjects, with very close concordance between the progression of cortical electroencephalographic arousal and systolic blood pressure (fig. 3).

\section{Discussion}

This study used a recently developed neural networkbased electroencephalographic analysis system to assess the changes in non-REM sleep depth that occur during Cheyne Stokes breathing. The results of this analysis were related to changes in breathing and arterial systolic blood pressure. This analysis found that sleep depth increased across the apnoeic phase of this breathing disturbance, reaching its nadir at or just preceding apnoea termination. Thereafter, sleep lightened as the subject moved towards "arousal". All subjects exhibited some lightening of sleep during their ventilatory phase and this was consistently associated with autonomic activation, indicated by a rise in systolic blood pressure. The rise in blood pressure occurred with a time course almost identical to that of the cortical electroencephalographic arousal described by the neural network technique.

\section{Sleep depth changes during Cheyne-Stokes breathing}

It was found that the depth of sleep increased across a Cheyne-Stokes apnoea to reach its nadir coincident with or just prior to the resumption of breathing (figures 2 and 3). There are few other studies with which to compare this result and no previous studies reporting sleep depth in detail during Cheyne-Stokes breathing. Several studies have noted the presence of intermittent visually evident electrocardiographic arousals during Cheyne-Stokes breathing $[3,4]$, but the inconsistency of this visually evident response has also been highlighted [15]. Indirect evidence for arousals being more consistently present comes from modelling studies, which suggest that repetitive arousal is probably necessary to sustain the periodicity of breathing [16]. It is interesting that, in this study, post-apnoeic arousal was a frequent phenomenon. This may be because the subjects chosen for study had relatively severe respiratory disturbances, as quantified by the apnoea/hypopnoea index.

ReEs et al. [9] reported changes in sleep depth during obstructive sleep apnoea using the fast Fourier transform (FFT). They found a rise in high-frequency spectral power during the second half of apnoea. Their methodology does not allow exact identification of the onset of this rise, (their FFT needed a window of half the duration of the apnoea to be informative). However, their results are consistent with the neural network analyses of obstructive sleep apnoea [10] and Cheyne-Stokes breathing presented here. Both of these analyses suggest arousal often begins in the last few seconds of an apnoea.

If the presence of a visible alpha rhythm on the EEG is used as the sole indicator of arousal, then arousal is characteristically a feature of the breathing phase of the cycle in both obstructive sleep apnoea and Cheyne-Stokes breathing. However, methods identifying subtle electroencephalographic frequency shifts not readily visually appreciated $[9,13,14]$ or use the entire electroencephalographic frequency spectrum $[13,14]$, confirm that some evidence of cortical activation can be detected in the absence of visual alpha rhythm arousal $[9,14]$. Thus, arousal is best seen as a continuous process not fully described by traditional categorical scoring [9].

The initiation of the cortical arousal process prior to apnoea termination is relevant to the possible mechanisms responsible for initiating arousal during sleep and breathing disorders. High ventilatory drive, which produces large falls in pleural pressure [17], is an effective arousal stimulus, and is probably important in arousal triggered by obstructive sleep apnoea. However, this is not an obvious explanation for the cortical and subcortical arousal beginning late in the apnoeic phase of Cheyne-Stokes breathing when respiratory efforts are characteristically absent. The finding of late apnoeic, pre-ventilatory, electroencephalographic and autonomic activation is in accord with animal data showing the onset of respiratory drive late in a central apnoea. Reduced animal preparations show the onset of phasic respiratory drive prior to the resumption of airflow $[18,19]$, and recent data from intact dogs infers 

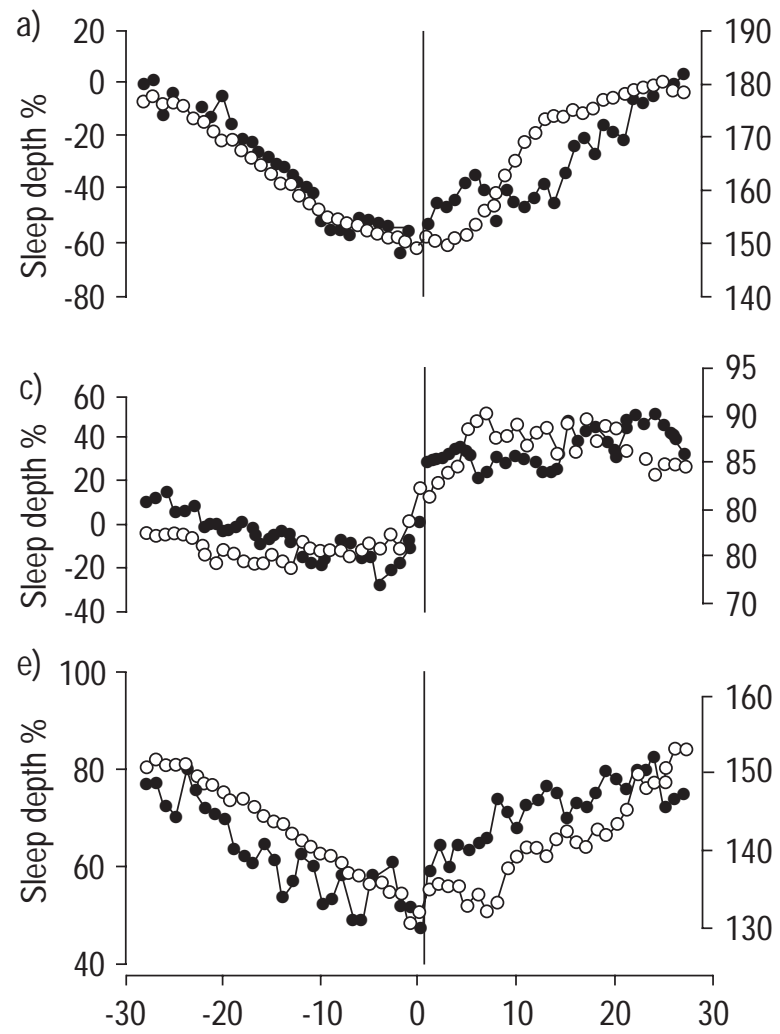

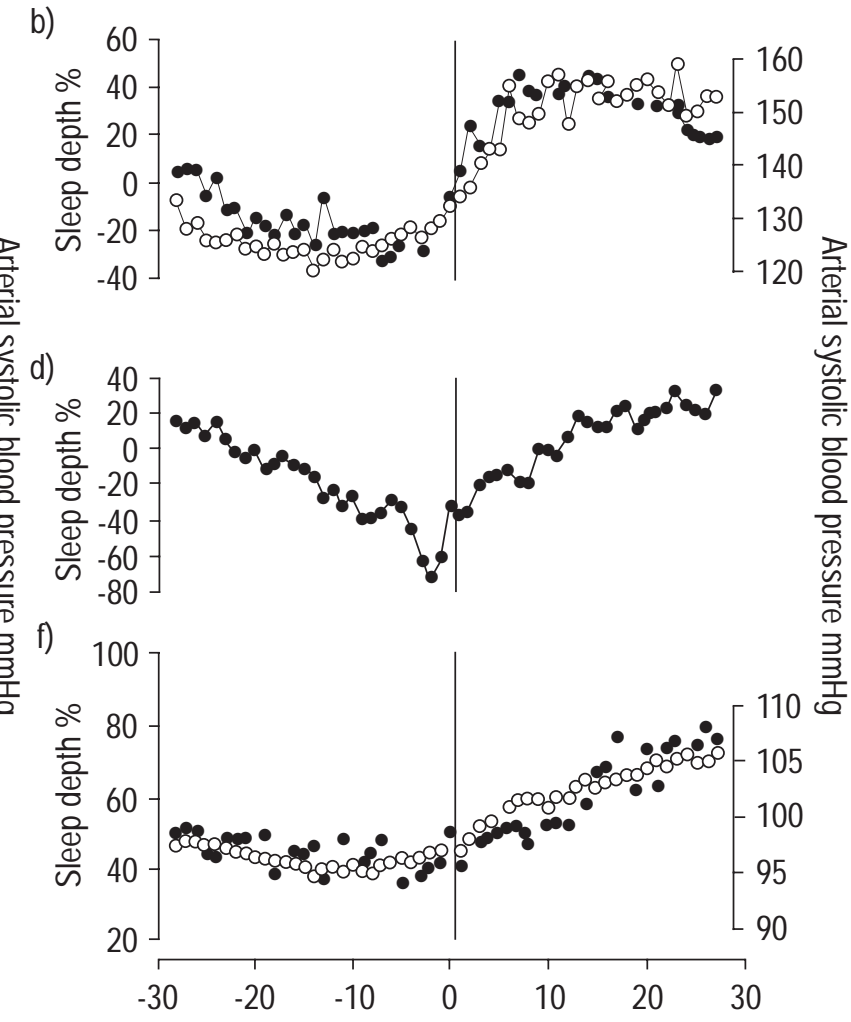

Time from apnoea break s

Fig. 3. - Pattern of change in neural network depth of sleep seen in Cheyne-Stokes breathing subjects: a) No. 1; b) No. 2; c) No. 3; d) No. 4; e) No. 5; f) No. 6. $\bullet$ : mean changes in depth of sleep; $\bigcirc$ : mean changes in systolic blood pressure. The onset of the first breath of the post-apnoeic ventilatory phase is indicated by the vertical bar.

late apnoeic respiratory centre output from a return of cardiac frequency variability with a respiratory periodicity before breathing resumes [19]. The results of this current study suggest that this respiratory activation is associated with a general autonomic activation and early cortical arousal, at least when the central apnoeas are occurring during sleep. Together these observations suggest that the late phase of central apnoea may be devoid of ventilation, but is not physiologically quiet.

It is difficult to draw other aetiological conclusions about the mechanisms of Cheyne-Stokes breathing from the present data. The cyclical nature of this breathing disturbance makes it difficult to infer whether any particular physiological disturbance is causal of, or caused by, another. For example, the relationship between blood pressure and the breathing cycle may be interpreted in either of two ways. For most of this paper blood pressure oscillations have been considered to be secondary to CheyneStokes breathing and the arousals it induces. However, it is possible that the cardiovascular responses could be driving the breathing cycle through cardiac output changes varying the lung-to-chemoreceptor delay time, and hence destabilizing feedback control.

A range of autonomic changes occur as part of arousal from sleep. These include blood pressure elevation [14], cardiac frequency increase (and hence baroreflex downregulation) [20], pupillary constriction and skin vasoconstriction [8], and are together termed the orienting reflex
[8]. These signals provide a potential marker for the brainstem elements of arousal. This study used systolic blood pressure to relate the progression of these responses to that of cortical electroencephalographic arousal and found that they move in synchrony. It is important to recognize that arousal is not the only factor capable of altering blood pressure and cardiac frequency during Cheyne-Stokes breathing. As in obstructive sleep apnoea, blood gas disturbances and diving reflex activation [21] may be relevant, although the mechanical influences of obstructed breathing on cardiac loading [21] are absent. In obstructive sleep apnoea, the bulk of the post-apnoeic blood pressure rise seems to be caused by an arousalrelated increase in total peripheral resistance due to muscle bed vasoconstriction [22, 23]. Since this study shows that some degree of electroencephalograph arousal is also a consistent feature of Cheyne-Stokes breathing, it seems likely that similar effects will be present in this disorder. Despite this probability, all that this study can directly conclude is that systolic blood pressure and the EEG move in close synchrony. It will require the exclusion of other factors before it becomes clear whether this relationship is truly aetiological or whether the two markers are simply coincident phenomena driven by the respiratory cycle.

This study has shown that the systolic blood pressure response which develops in Cheyne-Stokes breathing has a time course similar to that of electroencephalographic 
arousal. This observation has not been developed by performing an event-by-event comparison to establish how many Cheyne-Stokes apnoeas terminating in an ASDA arousal also terminate in a neural network event. This is because of the practical difficulties involved in defining a discreet "event" from the neural network trace. Events on this trace occur across the full continuum between little evident electroencephalographic change and obvious arousal. This is further complicated by discrete arousal-induced electroencephalographic events (particularly atypical K-complexes) which occur superimposed on the EEG during early arousal initiation. These events render the onset of arousals very volatile on the neural network trace, and, hence, the authors have found that a clean definition of arousal and its duration, essential for an event by comparison with ASDA events, virtually impossible. This problem is illustrated by work in obstructive sleep apnoea in which, in order to perform an event-by-event comparison with ASDA arousals, it was necessary to use a derivative of the rate of descent into sleep after the termination of the arousal [24]. It is this problem of variability during arousal onset which is also the reason for using an averaging technique across many breathing cycles in this analysis. This averaging process means that the exact point at which arousal is initiated may be blurred. For this reason the terminology "at or just before the resumption of breathing" has been chosen without being more exact.

\section{Conclusion}

This study reports the second-by-second changes in sleep depth during Cheyne-Stokes breathing. Using this method, sleep is seen to deepen across the apnoeic period, with the nadir in sleep depth occurring at or just before the resumption of breathing. Thereafter, arousal progresses during the ventilatory phase. These cortical electroencephalographic changes are very closely mirrored by changes in arterial systolic blood pressure, suggesting that, in this disorder, arousal may progress in the brainstem and cortex in synchrony.

\footnotetext{
Acknowledgements. The authors wish to thank S. Roberts for his involvement with the early data analysis.
}

\section{References}

1. Hanly PJ, Millar TW, Steljes DG, Baert R, Frais MA, Kryger MH. Respiration and abnormal sleep in patients with congestive heart failure. Chest 1989; 96: 480-488.

2. Findley LJ, Zwillich CW, Ancoli-Israel S, Kripke D, Tisi G, Moser KM. Cheyne-Stokes breathing during sleep in patients with left ventricular heart failure. South Med $J$ 1985; 78: 11-15.

3. Baylor D, Tayloe, Owen D, Sanders C. Cardiac failure presenting as sleep apnea. Elimination of apnoea following medical management of cardiac failure. Chest 1988; 94: $1298-1300$.

4. Dowdell WT, Javaheri S, McGinnis W. Cheyne-Stokes respiration presenting as sleep apnoea syndrome. Am Rev Respir Dis 1990; 141: 871-879.

5. Rechtschaffen A, Kales A. A manual of standardised ter- minology, techniques and scoring system for sleep stages of human subjects. Washington DC: National Institutes of Health, Publication No. 204; 1968.

6. Bonnet M, Carley D, Carskadon M, et al. EEG arousals: scoring rules and examples. A preliminary report from the Sleep Disorders Atlas Task Force of the American Sleep Disorders Association. Sleep 1992; 15: 173-184.

7. Martin SE, Wraith PK, Deary IJ, Douglas NJ. The effect of nonvisible sleep fragmentation on daytime function. Am J Respir Crit Care Med 1997; 155: 1596-1601.

8. Johnson LC, Lubin A. The orienting reflex during waking and sleeping. Electroencephalog. Clin Neurophysiol 1967; 22: $11-21$

9. Rees K, Spence DPS, Earis JE, Calverley PMA. Arousal responses from apnoeic events during non rapid-eyemovement sleep. Am J Respir Crit Care Med 1995; 152: 1016-1021.

10. Stradling JR, Davies RJO. Is it necessary to record sleep. Sleep 1996; 19: 251-25.

11. Pack AI, Cola MF, Goldszmidt A, Ogilvie MD, Gottschalk A. Correlation between oscillations in ventilation and frequency content of the electroencephalogram. $J$ Appl Physiol 1992; 72: 985-992.

12. Parati G, Casadei R, Groppelli A, Di Rienzo M, Mancia G. Comparison of finger and intra-arterial blood pressure monitoring at rest and during laboratory testing. Hypertension 1989; 13: 647-655.

13. Pardey J, Roberts S, Tarassenko L, Stradling J. A new approach to the analysis of the human sleep-wakefulness continuum. J Sleep Res 1996; 5: 201-210.

14. Davies RJO, Belt PJ, Robert SJ, Ali NJ, Stradling JR. Artenal blood pressure responses to graded transient arousal from sleep in normal humans. J Appl Physiol 1993; 74: $1123-1130$.

15. Trinder J, Merson R, Rosenburg J, Fitzgerald F, Kleiman J, Bradley TD. Effects of arousal on blood pressure fluctuations in patients with Cheyne-Stokes respiration and heart failure. Am J Respir Crit Care Med 1977; 155: 4: A776.

16. Khoo MCK, Gottschalk A, Pack AI. Sleep-induced periodic breathing and apnoea: a theoretical study. $J$ Appl Physiol 1991; 70: 2014-2024.

17. Gleeson K, Zwillich CW, White DP. The influence of increasing ventilatory effort on arousal from sleep. Am Rev Respir Dis 1990; 142: 295-300.

18. Batsel HL. Activity of bulbar respiratory neurons during passive hyperventilation. Exp Neurol 1967; 19: 357-37.

19. Horner RL, Brooks D, Kozar LF, Gan K, Phillipson EA. Respiratory-related heart rate variability persists during central apnoea in dogs: mechanisms and implications. $J$ Appl Physiol 1995; 78: 2003-2013.

20. Smyth HS, Sleight P, Pickering GW. Reflex regulation of arterial pressure during sleep in man. Circ Res 1969; 14: 109-121.

21. Davies RJO, Stradling JR. Acute effects of obstructive sleep apnoea. Br J Anaesth 1993; 1: 725-729.

22. Garpestad E, Katayama H, Parker JA, et al. Stroke volume and cardiac output decrease at termination of obstructive apneas. J Appl Physiol 1992; 73: 1743-1748.

23. Anand A, Taylor JA, Weis A, Weiss JW. The neurocirculatory changes following release of voluntary apnea in hypoxemic waking normal humans. Am $J$ Respir Crit Care Med 1997; 155: 4: A678.

24. Bennett LS, Stradling JR, Barbour CJ, Davies RJO. Cortical EEG arousal detection from a neural network (NN) analysis. Am J Respir Crit Care Med 1997; 155: 4: A775. 